\title{
Quark-Parton Model and Relativistic Quantum Mechanics
}

\author{
Boris Kostenko ${ }^{1, \star}$ \\ ${ }^{1}$ Joint Institute for Nuclear Research, Dubna, Russian Federation
}

\begin{abstract}
An attempt to treat the asymptotic freedom and the quark confinement as a self-consistent problem in the framework of relativistic quantum mechanics is realized. It is shown that the confinement of quarks induces a change of their helicities together with a simultaneous alteration of orbital momenta, so that the total angular momentum of each quark is conserved. This observation may cast light on the so-called proton spin puzzle after some additional numerical estimations.
\end{abstract}

\section{Introduction}

The Dirac field of a free quark polarized at rest along z-axis may be written in the form

$$
\psi=\sqrt{\frac{E+m}{2 m}}\left(1,0,0, \frac{p}{E+m}\right)^{T}
$$

in a reference frame moving along the direction of polarization. Using the probabilistic quantum mechanical interpretation [1] of the separate components of $\psi$, it is easy to check that the electric charge and the $z$-component of the quark spins are conserved after transformation into the moving frame. Of course, the quarks in a nucleon are not free and that can change drastically the picture. In particular, virtual excited states of quarks may appear in the bound state. Short-living in the rest frame, they become very long-living in the high-speed frame. We may even consider them as real excitations after transformation to the infinite momentum frame which underlies the parton picture of the nucleon structure. Just as the excited states of the hydrogen atom are described taking into account corresponding physical symmetries, the almost real excitations of a nucleon may be naturally treated in the same way. In contrast to the hydrogen atom which is characterized by spherical symmetry, the states of quarks in a relativistic nucleon should be axially symmetric relative to the direction of its motion. This fact brings into play the projection of the orbital angular momentum $L_{z}^{i}$ of the quarks on the symmetry axis. Indeed, if we admit the possibility of the observation of excited states of the nucleon (e.g., in the deep-inelastic lepton scattering on it) we should expect that the total helicity of the quarks $\sum S_{z}^{i}$ in the nucleon is not equal to the projection of nucleon's spin along the direction of movement. Only the total angular momentum, $\sum\left(S_{z}^{i}+L_{z}^{i}\right)$, may be constant. Below we shall consider these general arguments in more detail and try to estimate a possible value of the effect.

\footnotetext{
^e-mail: bkostenko@jinr.ru
} 


\section{Free partons in the nucleon}

Following the parton model which considers the quarks as free particles and taking into account the axial symmetry of their state, we write the Dirac equation for free fermions in cylindrical coordinates,

$$
\left[i\left(\gamma^{r} \frac{\partial}{\partial r}+\gamma^{\varphi} \frac{\partial}{r \partial \varphi}+\gamma^{z} \frac{\partial}{\partial z}\right)+\gamma^{0} E-m\right] \psi(r, \varphi, z ; E)=0 .
$$

Here $^{1}$

$$
\gamma^{r}=\left(\begin{array}{cc}
0 & \sigma^{r} \\
-\sigma^{r} & 0
\end{array}\right), \quad \gamma^{\varphi}=\left(\begin{array}{cc}
0 & \sigma^{\varphi} \\
-\sigma^{\varphi} & 0
\end{array}\right), \quad \gamma^{z}=\left(\begin{array}{cc}
0 & \sigma^{z} \\
-\sigma^{z} & 0
\end{array}\right), \quad \gamma^{0}=\left(\begin{array}{cc}
I & 0 \\
0 & -I
\end{array}\right),
$$

and

$$
\sigma^{r}=\left(\begin{array}{cc}
0 & e^{-i \varphi} \\
e^{i \varphi} & 0
\end{array}\right), \quad \sigma^{\varphi}=\left(\begin{array}{cc}
0 & -i e^{-i \varphi} \\
i e^{i \varphi} & 0
\end{array}\right), \quad \sigma^{z}=\left(\begin{array}{cc}
1 & 0 \\
0 & -1
\end{array}\right), \quad I=\left(\begin{array}{ll}
1 & 0 \\
0 & 1
\end{array}\right) .
$$

The substitution

$$
\psi=\left(\begin{array}{c}
q(r, \varphi) \\
\bar{q}(r, \varphi)
\end{array}\right) e^{i p_{z} z}
$$

separates the wave functions of the quarks and antiquarks and reduces the Dirac equation to the system of two partial differential equations,

$$
\left\{\begin{array}{l}
(E-m) q+\left[i\left(\sigma^{r} \frac{\partial}{\partial r}+\frac{\sigma^{\varphi}}{r} \frac{\partial}{\partial \varphi}-\sigma^{z} p_{z}\right)\right] \bar{q}=0, \\
(E+m) \bar{q}+\left[i\left(\sigma^{r} \frac{\partial}{\partial r}+\frac{\sigma^{\varphi}}{r} \frac{\partial}{\partial \varphi}-\sigma^{z} p_{z}\right)\right] q=0 .
\end{array}\right.
$$

From the second equation of the system, we get $\bar{q}$ in terms of $q$ and substitute the result into the first one. Thereby we obtain

$$
\left(\frac{\partial^{2}}{\partial r^{2}}+\frac{1}{r} \frac{\partial}{\partial r}+\frac{1}{r^{2}} \frac{\partial^{2}}{\partial \varphi^{2}}\right) q+p_{\perp}^{2} q=0
$$

where $p_{\perp}=\sqrt{E^{2}-\left(p_{z}^{2}+m^{2}\right)}$ is the transverse momentum of the quark. The separation of the variables by means of the substitution $q(r, \varphi)=u(r) e^{i n \varphi}$, where $n$ is an integer due to the single-valuedness of $q(r, \varphi)$, leads to the Bessel equation,

$$
\frac{\partial^{2} u}{\partial r^{2}}+\frac{1}{r} \frac{\partial u}{\partial r}-\frac{n^{2}}{r^{2}} u+p_{\perp}^{2} u=0 .
$$

Its solutions which are finite at $r=0$ are the Bessel functions of the first kind, $u(r) \sim J_{n}\left(p_{\perp} r\right)$. The corresponding wave functions of the quarks in the transverse plane, $q(r, \varphi)=u(r) e^{i n \varphi}$, are eigenstates of the $z$-component of the orbital momentum operator $\hat{L}_{z}=-i \partial / \partial \varphi$. In principle, the wave functions of this type can describe the orbital momentum contribution to the total helicity of the proton. However, they cannot describe the localization of the quarks inside the nucleon because $J_{n}\left(p_{\perp} r\right)$ have oscillating asymptotics damped as $1 / \sqrt{p_{\perp} r}$ at long distances. Since $\int_{0}^{r_{N}} u^{2}(r) r d r<\int_{r_{N}}^{\infty} u^{2}(r) r d r=\infty$ these solutions describe "wave functions" of quarks localized mainly outside the nucleon.

\footnotetext{
${ }^{1}$ The expressions for $\gamma$-matrices in cylindrical coordinates can be found taking into account the vectorial form of their transformations, $\gamma^{s}=\gamma^{i} \partial x^{s} / \partial x^{i}$.
} 


\section{Confined partons in the nucleon}

Here we consider a model describing the quark confinement within an external cylindrically symmetric static potential $V(r)$. Now the Dirac equation will involve the expression $E-V(r)$ instead of $E$. Then the equation (1) will undergo a change too, and instead of it we find

$$
\left(\frac{\partial^{2}}{\partial r^{2}}+\frac{1}{r} \frac{\partial}{\partial r}+\frac{1}{r^{2}} \frac{\partial^{2}}{\partial \varphi^{2}}+p_{\perp}^{2}\right) q(r, \varphi)+i \sigma^{r} \frac{-d V / d r}{E-V+m}\left[i\left(\sigma^{r} \frac{\partial}{\partial r}+\frac{\sigma^{\varphi}}{r} \frac{\partial}{\partial \varphi}\right)-\sigma^{z} p_{z}\right] q(r, \varphi)=0 .
$$

As compared to (1), an additional term proportional to the force $-d V / d r$ appears. The key observation explaining the mechanism of the quark spin flipping is as follows. The additional term contains the matrix

$$
\sigma^{r} \sigma^{z}=-i \sigma^{\varphi},
$$

which operates on the quark state with the positive helicity in the following way

$$
-i \sigma^{\varphi}\left(\begin{array}{l}
1 \\
0
\end{array}\right)=\left(\begin{array}{c}
0 \\
e^{i \varphi}
\end{array}\right)
$$

Thus, due to it, the projection of the spin changes its sign, the value of the orbital momentum increases by the unit and the total angular momentum remains constant,

$$
\left(S_{z}+L_{z}\right) q=\frac{1}{2} q, \quad\left(S_{z}+L_{z}\right) q^{\prime}=\frac{1}{2} q^{\prime}, \quad q=(1,0)^{T}, \quad q^{\prime}=-i \sigma^{\varphi} q .
$$

Now the separation of the $r$ and $\varphi$ variables is done through the substitution

$$
q(r, \varphi)=\left(\begin{array}{c}
u_{+}(r) e^{i n \varphi} \\
-i u_{-}(r) e^{i(n+1) \varphi}
\end{array}\right)
$$

where $u_{+}$and $u_{-}$are the wave functions of the quarks with positive and negative helicities. For the case $n=0$, which indicates that there are no orbital excitations in the region of the asymptotic freedom and all of them appear only due to the confinement force, we arrive at the system

$$
\left\{\begin{array}{l}
\left(\frac{\partial^{2}}{\partial r^{2}}+\frac{1}{r} \frac{\partial}{\partial r}+p_{\perp}^{2}\right) u_{+}(r)-\frac{d V / d r}{E-V+m}\left[\frac{\partial u_{+}(r)}{\partial r}+p_{z} u_{-}(r)\right]=0, \\
\left(\frac{\partial^{2}}{\partial r^{2}}+\frac{1}{r} \frac{\partial}{\partial r}+p_{\perp}^{2}-\frac{1}{r^{2}}\right) u_{-}(r)-\frac{d V / d r}{E-V+m}\left[\left(\frac{\partial}{\partial r}+\frac{1}{r}\right) u_{-}(r)+p_{z} u_{+}(r)\right]=0 .
\end{array}\right.
$$

We have assumed for $V(r)$ a realistic confinement potential found in the framework of lattice QCD in [2]. Following the parton model, we suppose that quarks are free in the vicinity of the nucleon center, $r<0.7 \mathrm{fm}$, and that the confinement force is manifested at larger distances in the region of the so-called gluon Y-string. Now the mathematical problem may be formulated as a Cauchy one for the system (2) with the initial conditions at $r_{0}=0.7 \mathrm{fm}$

$$
u_{+}\left(r_{0}\right)=J_{0}\left(p_{\perp} r_{0}\right), \quad u_{+}^{\prime}=J_{0}{ }^{\prime}\left(p_{\perp} r_{0}\right), \quad u_{-}\left(r_{0}\right)=0, \quad u_{-}^{\prime}=0 .
$$

The Runge-Kutta-Fehlberg algorithm recommended by MAPLE (rkf45 procedure) has not succeeded in finding a numerical solution compatible with the confinement conditions $u_{+},\left.u_{-}\right|_{r \rightarrow \infty} \rightarrow 0$. The following method has been applied instead. The confinement potential is written in the laddershaped form, so that $d V / d r=\Delta V\left(r_{i}\right) \delta\left(r-r_{i}\right)$. Everywhere, apart from points $r_{i}$, the system (2) results in two Bessel equations for $u_{+}$and $u_{-}$separately. Their solutions for $p_{\perp}^{2}>0$ are $u_{+}(r)=A_{1} J_{0}\left(p_{\perp} r\right)+A_{2} Y_{0}\left(p_{\perp} r\right), u_{-}(r)=B_{1} J_{1}\left(p_{\perp} r\right)+B_{2} Y_{1}\left(p_{\perp} r\right)$, where $J_{i}$ and $Y_{i}$ are Bessel functions of the 1 st and 2nd kind, correspondingly. Similarly, for $p_{\perp}^{2}<0$ we have solutions expressed in terms of modified Bessel functions of the 1st and 2nd kind, $u_{+}(r)=C_{1} I_{0}\left(\left|p_{\perp}\right| r\right)+C_{2} K_{0}\left(\left|p_{\perp}\right| r\right)$, $u_{-}(r)=D_{1} I_{1}\left(\left|p_{\perp}\right| r\right)+D_{2} K_{1}\left(\left|p_{\perp}\right| r\right)$. 


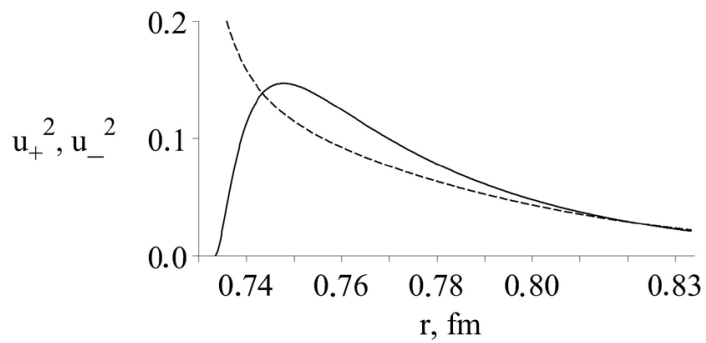

Figure 1. Quarks' wave functions squared corresponding to positive and negative helicities in the region of sub-barrier penetration (dashed and solid lines, respectively). They were calculated for $A_{2}, B_{2}$, $C_{1}, D_{1}=0$ (see text). The total polarization of the quarks is negative and equal to -0.0144 in the interval of radii shown in the figure.

\section{Computing algorithm and results}

The idea of our numerical solution is to join the above quoted Bessel functions by imposing on them matching conditions at points $r_{i}$ to satisfy the system (2). The conditions turn out to be

$$
\left\{\begin{array}{l}
\Delta u_{+}^{\prime}\left(r_{i+1}\right)=\frac{\Delta V\left(r_{i}\right)}{E-V\left(r_{i}\right)+m}\left(u_{+}^{\prime}\left(r_{i}\right)+p_{z} u_{-}\left(r_{i}\right)\right), \\
\Delta u_{-}^{\prime}\left(r_{i+1}\right)=\frac{\Delta V\left(r_{i}\right)}{E-V\left(r_{i}\right)+m}\left(u_{-}^{\prime}\left(r_{i}\right)+\frac{u_{-}\left(r_{i}\right)}{r_{i}}+p_{z} u_{+}\left(r_{i}\right)\right)
\end{array}\right.
$$

and can be found from (2) after neglecting the contribution of all finite terms at $r=r_{i}$. If all $A_{i}, B_{i} \neq 0$ we may impose some additional conditions. For example, for $u_{+}(r)$ one of the additional conditions in the region $p_{\perp}^{2}>0$ may be taken in a form

$$
A_{1 i} J_{0}\left(p_{\perp i} r_{i}\right)+A_{2 i} Y_{0}\left(p_{\perp i} r_{i}\right)=A_{1 i-1} J_{0}\left(p_{\perp i-1} r_{i-1}\right)+A_{2 i-1} Y_{0}\left(p_{\perp i-1} r_{i-1}\right) .
$$

Similar condition for $u_{-}(r)$ may be obtained by substitutions $A \rightarrow B$ and $J_{0} \rightarrow J_{1}, Y_{0} \rightarrow Y_{1}$. Analogues conditions may be written in the region $p_{\perp}^{2}<0$. This mathematical trick leads to constant solutions despite $K_{i}\left(p_{\perp} r\right), Y_{i}\left(\left|p_{\perp}\right| r\right) \rightarrow \infty$ at $\left|p_{\perp}\right| \rightarrow 0$. They can describe behavior of the wave functions of quarks in the neighborhood of the point $r_{\text {stop }}$ at which $p_{\perp}(r)=0$ and, thus, connect the classically accessible region (CAR) with the region of subbarrier penetration (SPR).

If one of the coefficients $A_{i}$ and one of $B_{i}$ is equal to zero the functions $u_{+}$and $u_{-}$themselves should undergo breaks. In this case we have two equations (2) for the determination of 2 nonzero coefficients. The same condition holds for the coefficients $C_{i}$ and $D_{i}$. The solutions of this type should be chosen according to the general Galerkin prescription to fulfil the necessary boundary conditions (of the finiteness here) at $r>r_{\text {stop. }}$.

Our results were obtained for $p_{z}=27.6 \mathrm{GeV}$ in agreement with the HERMES experiment [3], $m=0$ according to the parton model, $p_{\perp}(r=0.7)=2 \mathrm{GeV}$ (may be adjusted). The calculations have shown that the confinement potential cannot stimulate significantly the quark helicity flipping in CAR, but the effect is striking in SPR (see Fig. 1). For an exact numerical estimation of the effect, it is necessary to solve a problem of the smooth matching the $\operatorname{rcf} 45$ solutions at a point near $r_{\text {stop }}$ in SPR where $u_{+}(r)$ still drops with the solutions of the Galerkin type described above.

\section{Acknowledgement}

The author thanks Gh. Adam, N.V. Makhaldiani and A.B. Pestov for numerous valuable remarks.

\section{References}

[1] J.D. Bjorken and S.D. Drell, Relativistic Quantum Mechanics (McGraw-Hill, New York, 1964)

[2] T.T. Takahashi, H. Matsufuru, Y. Nemoto, and H. Suganuma, Phys. Rev. Lett. 86, 18 (2001)

[3] A. Airapetian et al., the HERMES Collaboration, Phys. Rev. D75, 12007 (2007) 